Recepción: 05/02/2018

Aceptación: 11/03/2019

Publicación: 05/04/2019

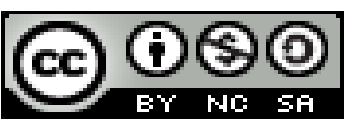

Ciencias económicas y empresariales

Artículo de revisión

\title{
Ámbitos de investigación de la carrera de administración de empresas: Emprendimientos estratégicos
}

\section{Fields of research in the career of business administration: Strategic ventures Áreas de pesquisa na carreira de administração de empresas: empreendimentos estratégicos}

\author{
Jorge Oswaldo Quevedo-Vázquez ${ }^{\mathrm{I}}$ \\ joquevedov@ucacue.edu.ec \\ Florencio Iván García-Álvarez ${ }^{\text {II }}$ \\ figarciaa@ucacue.edu.ec \\ Juan Diego Ochoa-Crespo ${ }^{\text {III }}$ \\ jdochoac@ucacue.edu.ec \\ Glenda E. Cabrera-Cárdenas ${ }^{\mathrm{III}}$ \\ glenda_cabrera81@hotmail.com
}

Correspondencia: joquevedov@ucacue.edu.ec

I Magíster en Administración de Negocios, Diploma Superior en Práctica Docente Universitaria, Economista, Licenciado en Economía y Finanzas, Docente Universidad Católica de Cuenca Sede Azogues- Unidad Académica de Administración, Sub Dirección de Posgrados, Cuenca, Ecuador.

II Magíster en Administración de Empresas Mención en Recursos Humanos y Marketing, Especialista en Docencia Universitaria, Ingeniero Comercial, Docente Universidad Católica de Cuenca, Cuenca, Ecuador.

III Magíster en Gestión Empresarial, Ingeniero Comercial, Docente Universidad Católica de Cuenca, Cuenca, Ecuador.

III Estudiante Universidad Católica de Cuenca-Sede Azogues, Cuenca, Ecuador. 


\section{Resumen}

El presente artículo se propuso evaluar el emprendimiento estratégico en el marco del ámbito de investigación de la carrera de administración de empresas. Para ello, considero una metodología de tipo teórica documental, en la que se empleó como fuente de información artículos científicos de revistas arbitradas nacionales e internacionales, disponibles en la web, así como la revisión de fuentes bibliográficas y no bibliográficas, estudios y/o antecedentes de la misma variable. Se utilizó un enfoque analítico - documental de la información a través de la triangulación de las teorías. El análisis de la información utilizo la hermenéutica para generar los resultados que permitieron crear el cuerpo de conclusiones. Entre sus resultados se destaca el hecho en la cual la investigación en materia de emprendimiento, desde un enfoque económico, considera al emprendedor como motor de desarrollo de la sociedad y eje del crecimiento económico. Se pudo concluir que el emprendimiento estratégico es un campo que ha ido evolucionado recientemente debido a las maneras de abordar su objeto de estudio desde el desarrollo humano y social, aportando evidencia teórica que ratifica dicha afirmación.

Palabras clave: Emprendimiento estratégico; emprendedor; carrera de administración.

\section{Abstract}

This article was proposed to evaluate the enterprise strategic within the framework of the field of business administration career research. For this reason, I consider a methodology of documentary theoretical type, which was used as a information source scientific articles in peerreviewed journals national and international, available on the web, as well as the review of bibliographic sources and not bibliographical, studies and/or history of the same variable. We used an analytical approach - documentary information through the triangulation of the theories. The analysis of the information use hermeneutics to generate results that allowed to create the body of conclusions. Its results include the fact in which research in the field of entrepreneurship, from an economic perspective, sees the entrepreneur as an engine of development of society and economic growth axis. One could conclude that strategic entrepreneurship is a field that has been recently evolved due to ways of addressing its object of study from the human and social development, providing theoretical evidence that confirms this statement. 
Key words: strategic, entrepreneurial venture, administration career.

\section{Resumo}

O presente artigo foi proposto para avaliar o empreendedorismo estratégico no âmbito do campo de pesquisa da carreira de administração de empresas. Para fazer isso, eu considero uma metodologia de tipo teórico documentário, que foi usado como uma fonte de informação artigos científicos de revistas nacionais e internacionais indexadas, disponíveis na web, bem como a revisão das fontes bibliográficas bibliográficos e não, estudos e / ou história da mesma variável. Uma abordagem analítico - documental da informação foi utilizada através da triangulação de teorias. A análise da informação utilizou a hermenêutica para gerar os resultados que permitiram criar o corpo de conclusões. Entre seus resultados, destaca-se o fato de que a pesquisa sobre empreendedorismo, do ponto de vista econômico, considera o empreendedor como o motor do desenvolvimento da sociedade e eixo de crescimento econômico. Concluiu-se que o empreendimento estratégico é um campo que evoluiu recentemente por causa das maneiras de abordar o objeto de estudo do desenvolvimento humano e social, proporcionando evidência teórica que confirma esta afirmação.

Palavras-chave: Empreendedorismo estratégico; empreendedor carreira de administração.

\section{Introducción}

El emprendimiento estratégico es un método para los administradores que les permiten conocer la empresa desde cero, también les sirve para buscar nuevas formas de innovación para el mejoramiento de dicho negocio o empresas, esto les ayuda a implementar nuevas formas de poder garantizar un avance para un mejor funcionamiento del recurso de la empresa. En el análisis del entorno en las organizaciones explica que se asimila a relaciones de causa-efecto y de impacto. Se estudian los rasgos generales de la actividad emprendedora generada por el sistema económico global. El emprendimiento empresarial, es una alternativa diferente a la ya conocida, que permite más libertades a quien la ejercen, el emprendimiento estratégico se define como la iniciativa de un individuo para desarrollar un proyecto de negocios o una idea en particular que genera ingresos, crear una microempresa o (PYMES). 
El emprendimiento es un campo que cada día va evolucionando mediante nuevas ideas, generaciones, innovación, tecnología, entre otras técnicas y estrategias que se aplica en las empresas para el mejoramiento de la calidad de vida de los empresarios y del entorno en general para lo cual se debe de aplicar la disciplina y demás valores que en la actualidad pocas personas lo aplican en la vida empresarial y de eso depende para tener éxito como emprendedor.

Sin ánimo de desconocer los grandes desarrollos académicos e investigativos en materia de creación de empresas y de puesta en marcha de ideas de negocios, es evidente la necesidad de explorar el emprendimiento académico evitando su reducción a conceptos como empresa, empresarismo o empresario.

Todo parece indicar que el emprendimiento resulta ser una de las fórmulas adoptadas para enfrentar las épocas de crisis, de cambio y de incertidumbre a las que se someten las distintas sociedades. Sin temor a la equivocación, en la actualidad todos los sectores de la sociedad y de la economía se convierten en semilleros del emprendimiento. Se camina franca y decididamente hacia una gestión integral y transdisciplinar del emprendimiento. Y es que su importancia no puede considerarse como un asunto menor. La contribución del emprendimiento al desarrollo económico se da vía la innovación y la convergencia en el mercado, llevando así a la efíciencia económica.

De allí que, desde mucho tiempo el emprendimiento ha estado presente en el mundo para el desarrollo tanto público como social, al tomar un emprendimiento se debe tener disciplina capacidad y carácter, la limitación del emprendimiento en el ámbito de los negocios en las escuelas y facultades de administración de empresas es la misma enseñanza la creación de empresas, en la actualidad la economía es la base emprendimiento. Con base a lo expuesto, este artículo tiene como finalidad evaluar el emprendimiento estratégico en el marco del ámbito de investigación de la carrera de administración de empresas.

\section{Desarrollo}

Ramírez, A. (2009) manifiesta que en el análisis del direccionamiento estratégico se identifica una diversidad de factores internos y externos del sector, que configuran de distintas formas la gestión o acción de la empresa y/o estrategia que busca el cumplimiento de objetivos de la organización. En su artículo enfoca el análisis de los factores externos o entorno, que influencia 
el desarrollo del direccionamiento estratégico como propósito principal. En el análisis del entorno se identifican una diversidad de factores (económico, social, político, tecnológico, demográfico, jurídico, cultural, geográfico, ecológico) que se manifiestan por medio de la cultura, el lenguaje, la relación con el medio, las relaciones inter organizacionales, las presiones sociales derivadas de la violencia, el desplazamiento forzado, la corrupción política, entre otros. Son escenarios que configuran el entorno organizacional, la empresa y su direccionamiento estratégico donde la gestión empresarial debe desarrollarse de manera holística, hermenéutica y ecléctica mediante la continua relación con su entorno, con el propósito de alcanzar resultados que conviertan toda la información del entorno en beneficios económicos y sociales para la organización, con la alternativa del crecimiento y sustentabilidad. Este discurso sobre la "Influencia del Entorno en el desarrollo del Direccionamiento Estratégico "es parte del proyecto "Modelo de Direccionamiento Estratégico para la Generación de Políticas de Crecimiento Empresarial”. En lo mencionado por el autor, este tiene un carácter explorativo y descriptivo que ayuda a tener ideas para alcanzar un direccionamiento estratégico y así lograr todos los objetivos, asimismo muestra algunas alternativas para llevar bien un emprendimiento. Esto da a conocer la influencia del entorno en la empresa que afecta directamente a la gestión por la intensidad de sus impactos.

Moreno, Z. (2017), expresa que se deben determinar acciones estratégicas para generar el emprendimiento social desde las universidades de administración privada para que el pensamiento estratégico y las acciones estratégicas permitan el emprendimiento social a través de Proyectos de Negocios y Acciones filantrópicas. El mismo autor, refiere que el "objetivo estratégico" tiene como finalidad determinar el emprendimiento en las universidades para que puedan ejercer una capacidad única y busque el mejor beneficio, en la que se pueden ejercer proyectos para la toma de decisiones futuras en pro del mejoramiento de dicha empresa o negocio sin hacer a un lado el enfoque principal.

Por su parte Merino, C. (2004) narra en su artículo que en este discurso se antoja como piedra angular el contexto del fenómeno emprendedor aludiendo a su influencia en condiciones de renovación de los sectores y la mejora de las condiciones socioeconómicas. En este caso, la búsqueda de un colectivo de emprendedores con mayor impacto ha llevado a un marco de emprendimiento de especial interés, a saber, las Nuevas Empresas de Base Tecnológica (NEBT) 
donde las expectativas de crecimiento y competitividad resultan de gran calado. Es de señalar, que el objetivo es buscar nuevas renovaciones para poder emprender con mayor impacto y que la prestación de servicios de esta permita poner de manifiesto algunos factores claves para fundamentar, diseñar y realizar una innovación que genere cambio que beneficien el crecimiento y desarrollo de un país.

Toca, C. (2010) manifiesta que la reiterada limitación del emprendimiento al ámbito de los negocios y la enseñanza del mismo en escuelas de negocios y facultades de administración de empresas, han negado muchas posibilidades para su aplicación y adopción en ámbitos diferentes como el público y social. En la actualidad los emprendimientos son de distinta índole: colectivos, vecinales, públicos y sociales, por lo que no existe justificación para excluir a organizaciones e individuos de las bondades que ofrece como característica y condición.

Valencia. (2017) abarcan sus ideales en las condiciones de globalización en los entornos competitivos que han modificado las características sociales, económicas y culturales asociadas a ellos. En consecuencia, el fenómeno del emprendimiento comenzó a ser popular no solo entre las personas del común, sino también entre los empresarios, para quienes el fenómeno es más conocido como emprendimiento empresarial. El reconocimiento y la popularidad alcanzados por el fenómeno se debe, principalmente, a los efectos positivos observados en la creación de valor para el cliente, al brindarle productos y servicios nunca antes pensados; sin embargo, también implica grandes cambios a nivel de cultura y estructura en la empresa, al exigir comportamientos como la flexibilidad, la toma de riesgos y el dinamismo. El entorno empresarial demanda que las empresas establecidas adopten estrategias emprendedoras como camino hacia el éxito.

Alizo, M (2012) da a entender el ámbito actual, cambiante, flexible y de supeditación tecnológica que genera necesidades de ajustes y adaptaciones de la ciencia a la disposición y uso de los requerimientos reales de la sociedad, de la que surge el concepto e importancia del tema del emprendimiento, como un componente fenomenológico sólido en los diversos sistemas. Los emprendedores direccionan la innovación, aceleran cambios estructurales en la economía, contribuyen a estabilizar el crecimiento auto sostenido del mercado nacional y mitigan la sobredemanda de empleos a nivel mundial, generando nuevas oportunidades al crear necesidades alternativas. 
También, García y col (2016) exponen que el entorno socio económico actual es objeto de transformaciones enriquecedoras. En este sentido, la Constitución Ecuatoriana en el artículo 283, establece que el sistema económico es social y solidario, e incluirá a los sectores cooperativistas, asociativos y comunitarios. Donde se asume que favorecer empresas pequeñas e individuales implica también profundizar en su estudio para comprender su funcionamiento e impulsar el éxito, con énfasis en el papel que juega en la economía de un país. En este contexto el emprendedor, como actor económico, sin ser una panacea económica, representa una palanca para la innovación y el crecimiento. El Plan del Buen Vivir tiene implícita la mejora del bienestar de la población y lograr una economía adaptada a las nuevas condiciones internas y externas donde sea posible el desarrollo y una buena calidad de vida, se plantea como una prioridad el apoyo a los emprendedores, al tener en cuenta las posibilidades concretas que en estas formas de gestión económica subyacen.

Játiva M. (2015), en su trabajo prevé realizar una revisión y recopilación de las diferentes posturas de algunos expertos, quienes se han preocupado por definir y concretar que es; emprendimiento e innovación. Para ampliar la comprensión de este tópico se presenta una exposición exploratoria del emprendimiento en el Ecuador correspondiente al año 2015. Sin tratar de cubrir precisamente todos los ámbitos o aspectos referidos al emprendimiento en el Ecuador, se ilustra de manera general los siguientes temas:

$\checkmark$ En dónde prefieren invertir los ecuatorianos

$\checkmark$ Cuánto invierten los ecuatorianos

$\checkmark$ Comparación de la TEA (Tasa Económicamente Activa) con los países símiles de la región

$\checkmark$ La evolución de la actividad emprendedora temprana TEA

$\checkmark$ El desarrollo por zona urbana y rural y;

$\checkmark$ Las principales características de los emprendedores, sexo, ocupación e ingresos.

El mismo autor, apunta que el Ecuador, es un país dónde las mayores inversiones para emprender son variadas, dependiendo de factores de dinero, espacio, recursos y demanda, entre otras. En 
este sentido, Boza, J. (2013), indica que el análisis crítico a la economía popular y solidaria puso de relieve su problema en especial los asociados a proceso de análisis de los emprendimientos que la integran, partiendo de ellos considera necesario diagnosticar el desempeño de estos como base para el diseño productivo que contribuya a estabilizar el crecimiento del mercado nacional, generando nuevas oportunidades al crear necesidades alternativas. Sin embargo, mundialmente el rol del emprendedor varía según el nivel de desarrollo de la economía. En el carácter desarrollado, el emprendimiento surge como una oportunidad, sustentada en la investigación, el conocimiento y la innovación. Destaca al carácter del emprendedor como una oportunidad para implementar nuevas estrategias donde el conocimiento y la participación de toda la organización den un buen fruto y así se puedan alcanzar las metas que se tienen propuestas.

Pico y col (2016), exponen que el emprendimiento es un campo que ha ido evolucionado recientemente debido a las maneras de abordar su objeto de estudio. Su análisis ha girado en torno a tres enfoques; económico, psicológico e institucional. Este tema sugiere que existe un vacío en los estudios del emprendimiento visto desde el desarrollo humano y social. El emprendimiento es un campo que ha ido evolucionando recientemente debido a las maneras de abordar su objeto de estudio que se debe analizar desde cuatro perspectivas que intervienen en la creación de las empresas, tales como individuos, organización, ambiente y proceso. Estas cuatro dimensiones interactúan entre sí, es decir no es posible analizar el fenómeno del emprendimiento si no se analizan en conjunto estas cuatro dimensiones, por lo tanto, no son mutuamente excluyentes.

Toca (2010) señala que el emprendimiento ha estado supeditado a la esfera empresarial mientras su desarrollo en otros ámbitos, como el público y el social, es notablemente incipiente, a pesar de los esfuerzos que se realizan en instituciones educativas de todo nivel, centros de desarrollo y agencias gubernamentales. En su documento propone una reflexión sobre la importancia del emprendimiento para el desarrollo eficiente y competitivo de las sociedades, tomando como punto de partida los diferentes conceptos que se han adoptado, a fin de resaltar su carácter transdisciplinaria. Posteriormente, revisa algunas experiencias para explorar nuevas posibilidades de aplicación y, por último, clama por una apertura a nuevas dimensiones de la vida en sociedad. 
Reconoce el emprendimiento no como disciplina formal sino como actividad transdisciplinaria, carente de una teoría universal y soportada mayoritariamente en teorías prestadas de la economía, la ingeniería, la historia económica y empresarial, la sociología, la administración y, recientemente, de la psicología. Constituye un tema en boga, por lo que los gobiernos intentan promoverlo, los individuos aspiran practicarlo, las organizaciones buscan desesperadamente recapturarlo y la academia aspira estudiarlo, teorías del equilibrio neoclásico, en las que atributos personales definen perfiles de emprendedores, teorías psicológicas, que reconocen el emprendimiento como un proceso que depende de la habilidad y la voluntad del individuo para promover acciones, teorías austriacas, cuyo supuesto es que los individuos poseen diferente información que conduce a que visualicen distintos valores en un bien o servicio dado. Siendo la generación de nuevas empresas y el desarrollo de mercados los más estudiados, es cuestión de todos examinarlo a partir de características individuales de los emprendedores.

Ramírez, A. (2009), en su análisis del entorno en las organizaciones explica que se asimila a relaciones de causa-efecto y de impacto, debido a que a través de éstas se opera el concepto de influencia del entorno y su incidencia en las acciones estratégicas de las organizaciones .El análisis de las variables del entorno constituye una orientación para la acción de las organizaciones, al identificar claramente la acción estratégica correspondiente para aumentar o disminuir la efectividad de la empresa, reflejándose en su direccionamiento estratégico por medio de la elaboración de productos y prestación de servicios. La influencia del entorno en la empresa configura el direccionamiento estratégico debido a que el carácter incluyente de la sociedad refleja su impacto en la gestión empresarial.

La influencia del entorno en la empresa impacta directamente en su gestión, y hace complejo su ejercicio, en determinadas ocasiones es difícil de gestionar por la intensidad de sus impactos, que motivan una gestión integral de los recursos, porque se altera y modifica directamente el direccionamiento estratégico.

En otra conceptualización el autor da a conocer que este tiene un carácter explorativo y descriptivo que ayuda a tener ideas para lograr un direccionamiento estratégico y así lograr todos los objetivos, este también muestra algunas alternativas para llevar buen un emprendimiento 
dando a conocer la influencia del entorno en la empresa que afecta directamente a la gestión por la intensidad de sus impactos.

Moreno, Z. (2017) expone como objetivo determinar las acciones estratégicas para generar el emprendimiento social de la administración privada, en la que expuso que el pensamiento estratégico y las acciones estratégicas son determinantes para el emprendimiento social a través de proyectos de negocios y acciones filantrópicas. De igual manera evidenció que la planificación y toma de decisiones son de carácter empírico sin desestimar el enfoque racional de pensamiento.

Duarte, F. (2013) dice que en general, son emprendedores quienes individual o grupalmente crean ideas de negocio. Sin embargo, existen también otros importantes actores empresariales denominados intraemprendedores, quienes son los empleados que trabajan dentro de las empresas. Otro enfoque los define como "caminantes del futuro", suelen llamarse intraemprendedores al directivo que crea nuevas empresas desde su responsabilidad dentro de la misma. También lo es aquel empleado que realiza mejoras o innovaciones en una firma en marcha. Las empresas requieren, pues, entender la problemática social que las rodea, para ello, tienen la oportunidad de servirse de la experticia de los emprendedores sociales, siendo este un desarrollo natural de cualquier economía, pero teniendo en cuenta los aspectos sociales e incluyentes de la sociedad, así como identificar las competencias del personal brindando oportunidades a todos.

En síntesis, el autor se refiere que circunstancialmente los problemas que se presenta en la empresa tienen que ser manejado por todo el grupo asociado que forma parte de ello, donde cada persona aporta con nuevas ideas para protagonizar las clases de manera natural y sin alterar algún resultado con opción de realizar innovación.

Marulanda, F. (2016) da a conocer en este sentido la generación de nuevas empresas que se ha convertido en un reto permanente para las diferentes instancias gubernamentales, por cuanto existe una necesidad de generar fuentes de empleo y riqueza de forma permanente, que coadyuven al mejoramiento de las condiciones socioeconómicas de las comunidades, en el entendido de que el emprendimiento es un motor de desarrollo. En este contexto, los emprendedores se convierten en un eje fundamental, puesto que son ellos los que identifican las oportunidades y actúan en pro de su aprovechamiento. 
Para Ramírez, A. (2017) expresa que, en el estudio de las motivaciones para emprender, se han utilizado diferentes clasificaciones, una de las más reconocidas es la que se hace de acuerdo con la fuente de ellas, según la cual pueden ser de tipo intrínseco o extrínseco. Las de tipo extrínseco son aquellas que vienen desde fuera del individuo. En este grupo se incluye la existencia en el entorno de facilitadores para la creación de empresas.

Santos, F y col (2013) validan que se estudia el papel que pueden desempeñar los emprendimientos sociales en la consecución de un sistema económico mundial más justo. Para ello, se comienza colocando de manifiesto los problemas esenciales observados en el sistema económico global y la influencia que sobre ellos tiene la separación existente entre ética y economía. Posteriormente, se estudian los rasgos generales de la actividad emprendedora generada por el sistema económico global para, a continuación, explicar la forma de un tipo específico de emprendimiento, el emprendimiento social, puede propiciar un cambio de paradigma que contribuya a un sistema económico global más justo.

Hidalgo y col. (2017), explican que los programas orientados hacia el campo de la administración de negocios en el Ecuador carecen, en muchos casos, del enfoque hacia el emprendimiento, y se sigue formando a los futuros administradores o creadores de empresas en carreras de corte administrativo tradicionales que no preparan a los futuros graduados para la identificación de necesidades de productos y servicios en la sociedad y sobre todo en la posibilidad de responder a estas necesidades mediante la identificación de oportunidades que sirvan de base para la innovación o la creación de nuevas empresas (Arteaga y Lasio, 2010).

En otras observaciones de los autores la formación del emprendiendo tiene especificado sus normativas en la formación de Pymes. En lo competente en el Ecuador, donde los obstáculos de los emprendimientos para su eficaz desarrollo son de no poseer una buena administración.

Sin embargo, si se tienen buenas metas y una excelente administración se tendrá éxito en su emprendiendo.

Játiva, M. (2015). Manifestó que los ecuatorianos prefieren invertir para el emprendimiento de los sectores de comercio al por mayor y al por menor; entre los que se puede destacar; 50.048 actividades de reparación de vehículos automotores; y, motocicletas, 14.712 actividades de 
alojamiento y servicios de comida, 7.535 industrias manufactureras y 7.466 otras actividades de servicios y 5.303 emprendimientos en información y comunicación. En relación a la cantidad de inversión, aproximadamente invierte USD 2.218 al iniciar un proyecto. En lo que tiene que ver con los requerimientos de dinero para financiar la adquisición de activos fijos, estos llegan a un valor medio de 5.071 dólares. En conclusión, la inversión total media requerida para arrancar un emprendimiento es de USD 7.300. El ciclo se compone de cinco etapas, inicia por la Motivación de crear un negocio nuevo, el estímulo de la creatividad, se concreta en un proceso de planificación, continúa con la búsqueda del financiamiento interno o externo por medio de las Instituciones financieras para concretar la puesta en marcha. (Wilson Araque, 2015)

La autora, indica que el Ecuador mantiene una actividad emprendedora temprana y prematura (TEA por sus siglas en inglés) en la parte alta, 33.6\%, ubicándose en el primer lugar entre los países de América Latina y el Caribe, le siguen en orden de magnitud de la TEA, Chile con $25.9 \%$, Colombia con $22.7 \%$, y Perú con $22.2 \%$. Chile y Perú muestran reducción en la actividad emprendedora en relación al 2014, mientras que ésta ha aumentado con respecto al año pasado para Colombia y Ecuador. (ESPAE, 2015)

\section{Metodología}

La investigación fue de tipo teórica documental, en la que se empleó como fuente de información artículos científicos de revistas arbitradas nacionales e internacionales, disponibles en la web, así como la revisión de fuentes bibliográficas y no bibliográficas, estudios y/o antecedentes de la misma variable. El diseño documental, según Hurtado (2010), permite revisar fuentes de información no vivas. Se utilizó un enfoque analítico - documental de la información a través de la triangulación de las teorías y posiciones expuestas por los diferentes autores que versaron la temática del emprendimiento desde el punto de vista de la investigación en la carrera de Administración de empresas. El análisis de la información utilizo la hermenéutica para generar los resultados que permitieron crear el cuerpo de conclusiones.

\section{Resultados}

Entre sus resultados, se pudo evidenciar que: 
- El emprendimiento ha sido abordado por diferentes autores, quienes bajo distintos enfoques han concebido el concepto de emprendimiento y el rol del emprendedor en el desarrollo. El primer enfoque, denominado enfoque económico, considera al emprendedor como motor de desarrollo de la sociedad y eje del crecimiento económico.

- En el enfoque institucional, el emprendimiento está determinado por factores institucionales que condicionan la creación de nuevas empresas, desde lo informal con el espíritu emprendedor y actitudes hacia el emprendimiento y desde lo formal con organismos y medidas de apoyo para la creación de empresas, trámites y costos, entre otros.

- La relación entre el emprendimiento y el desarrollo humano genera oportunidades para investigar y profundizar sobre el tema, conociendo que siempre el emprendimiento ha estado presenta en la cultura empresarial del hombre en pro del desarrollo de lo público y social.

- Para tener un buen emprendimiento se debe tener disciplina y capacidad para reconocerlo no como disciplina formal sino como actividad empresarial que se apoya y desarrolla en el crecimiento.

- El desequilibrio que causa la exclusión, el sufrimiento humano, la creación del valor social, la innovación social, la pro actividad y los riesgos influyen en el emprendimiento empresarial.

- La operación-evaluación de un negocio, permite generar planteamientos donde la necesidad o la característica social han de estar presentes en cada idea durante la construcción de una empresa con sentido social

- Los procesos de emprendimientos deben tener en cuenta que el sistema económico global no es justo o no lo es tanto, pero que tienen que acostumbrarse y trabajar de una manera adecuada para acoplarse al sistema monetario económico y social de un país, cuidad o localidad. 


\section{Conclusiones}

El emprendimiento estratégico es un campo que ha ido evolucionado recientemente debido a las maneras de abordar su objeto de estudio desde el desarrollo humano y social, aportando evidencia teórica que ratifica dicha afirmación.

No es posible analizar el fenómeno del emprendimiento si no se analizan en conjunto los cuatros perspectivas que intervienen en la creación de las empresas; individuos, organización, ambiente y proceso, dado que interactúan entre sí durante cualquier proceso de emprendimiento.

El emprendimiento ha estado supeditado a la esfera empresarial mientras su desarrollo en otros ámbitos, como el público y el social, es notablemente incipiente, a pesar de los esfuerzos que se realizan en instituciones educativas de todo nivel, centros de desarrollo y agencias gubernamentales.

Los emprendimientos son de distinta índole: colectivos, vecinales, públicos y sociales. Todo parece indicar que el emprendimiento resulta ser una de las fórmulas adoptadas para enfrentar las épocas de crisis.

En el ámbito actual, cambiante y flexible el emprendimiento ayuda a estabilizar el crecimiento auto sostenido del mercado nacional, para lo cual se debe tener en cuenta que al momento de pasar al mercado nacional está proporcionando estabilidad económica en su empresa.

\section{Referencias Bibliográficas}

Alizo M. (2012). Factores clave de gestión económica estratégica del emprendimiento tipo Pyme, vinculados al mercado en Venezuela. Caracas, Venezuela

Boza J. (2013). Diagnóstico estratégico de emprendimientos de economía popular y solidaria en ecuador. Ecuador

Boza J y Manjarez, N. (2017). Diagnóstico estratégico de emprendimientos de economía popular y solidaria en ecuador. La Habana, Cuba

García, González .y Murillo (2016). Estudio sobre los emprendimientos de la economía simple en el Ecuador. - Ecuador. 
Hidalgo L., Trelles I., Catro A., y Loor B. (2017). Estrategias de compresión lectora: enseñanza y evaluación en el ecuador primaria. Ecuador.

Hurtado J (2012). “El proyecto de investigación Comprensión holística de la metodología y la investigación”. 7ma Edición. Quirón.

Játiva M. (2015). Emprendimiento e innovación una oportunidad en el Ecuador. Ecuador.

Merino C. (2004) dirección estratégica del conocimiento en el contexto de las nuevas empresas de base tecnológica. Universidad Autónoma de Madrid- España Madrid

Moreno Z. (2017) consideraciones acerca del emprendimiento estratégico

Pico A, Del Río Cortina J, Simancas J (2016). ¿El Emprendimiento como Estrategia para el Desarrollo $\quad$.. $\quad$ - $\quad$ Dialnet. Recuperado de: https://dialnet.unirioja.es/descarga/articulo/6069704.pdf

Ramírez A. (2009) Influencia del Entorno en el desarrollo del direccionamiento estratégico. Colombia.

Ramírez A. (2017). Nuevas perspectivas para entender el emprendimiento empresarial. Barranquilla, Colombia

Santos F., Barroso M., Guzmán, C (2013). La economía global y los emprendimientos sociales. Huelva, España

Toca C. (2010), Educación emprendedora: Estado del arte . Recuperado de: http://www.scielo.org.pe/scielo.php?script=sci_arttext\&pid=S2307-79992017000200010.

Valencia (2017) Consideraciones acerca del emprendimiento estratégico como síntesis funcional de la identificación de oportunidades y la creación de ventajas competitivas. Revista Virtual Universidad Católica del Norte. 